\title{
Prothesenversorgung nach Amputationen an der unteren Extremität
}

\author{
Bernd Sibbel, Detlev Kokegei
}

\section{Zusammenfassung}

Jede auch noch so geringfügige Amputation einer Extremität bedeutet für den Betroffenen einen erheblichen Eingriff in seine körperliche Unversehrtheit. Den Defekt auszugleichen und $\mathrm{zu}$ erwarten, dass ein prothetischer Ersatz die natürlichen Funktionen des Fußes oder des Kniegelenkes gleichwertig ersetzen kann, ist auch heute mit dem Einsatz modernster Materialien oder Passteile nicht möglich. Der vorliegende Artikel soll das interdisziplinäre Behandlungsteam über die prothetischen Versorgungsmöglichkeiten nach einer notwendigen Amputation informieren, um dem Amputierten eine bestmögliche Versorgung zukommen zu lassen. Dem Arzt kommt dabei die wichtigste Aufgabe zu. Er ist über die Verordnung und Abnahme des Hilfsmittels hinaus verantwortlich für die Gesamtkoordination aller Behandlungsmaßnahmen und somit auch für das Ergebnis der Rehabilitation. Wichtig ist, dass alle Maßnahmen zur Rehabilitation des Amputierten so früh wie möglich beginnen. Hierzu gehört die Anleitung zur korrekten Lagerung des Stumpfes bis hin zur Ödemkontrolle über Kompression oder auch über Hinweise zur Pflege der Haut. Zur Entgegenwirkung möglicher Kontrakturen sind postoperative physiotherapeutische Bewegungsübungen oder Muskeltraining indiziert. Eine Interimsprothese ermöglicht dabei gleichzeitig die ersten Steh- und auch Gehversuche. In diesen Abschnitt der Rehabilitation fällt auch die Verordnung des Arztes in Zusammenarbeit mit dem Orthopädietech- niker über die geeignete Form der prothetischen Versorgung. Das sich ergebende Anforderungsprofil an eine Prothese erfordert in erster Linie die einfache Handhabung des Hilfsmittels. Dazu gehört, dass der Patient dieses ohne Hilfestellung an-, vor allem aber auch selbstständig wieder ablegen kann.

Weitere Anforderungen sind:

- Sichere Steh- und Gehfähigkeit

- Geringe Masse (Eigengewicht; Gewicht der Passteile, vor allem des Fußes)

- Leichte Einleitung der Schwungphase und angepasste Schwungphasensteuerungen beim Einsatz von Kniepassteilen

- Angepasste Fußeigenschaften (Fersenauftritt, Abrollung, Ballenlösung; Pro- und Supination)

- Altersspezifischer Aufbau (Stumpfsituation beachten)

- Wiederherstellung des äußeren Erscheinungsbildes.

\section{Prosthesis Fitting after Amputations on the Lower Limb}

For the patient, every, even most minor amputation on a limb represents an appreciable attack on his/her bodily integrity. It is not possible to compensate for the defect and to expect that a prosthetic replacement will completely substitute for the natural function of the foot or knee joint even with the use of the most modern materials and/or fittings. The present article is intended to inform the management team about the prosthetic possibilities after a necessary amputation, in order to provide the amputee with the best available management. Here the physician has the most important tasks. He/ she is not only responsible for prescribing and accepting the prosthetic aid but also for the total coordination of all therapeutic measures and thus for the success of any rehabilitation. It is most important that all measures for rehabilitation of the amputee are started as early as possible. Among these are instructions for correct position of the stump through to checking for edema and compression or also advice for care of the skin. Postoperative physiotherapeutic movement and muscle training is indicated to counteract any possible contractures. An interim prosthesis can thereby facilitate the first standing and walking attempts. This stage of rehabilitation includes the physician's prescription of a suitable form of prosthetic management in cooperation with the orthopedic technician. The thus derive requirement profile of the prosthesis demands in the first instance the simple handling of the apparatus. In this way it must be possible for the patient to apply and remove the prosthesis without any external help.

Further requirements are:

- Safe ability to stand and walk

- Low weight (own weight, weight of fitted parts, especially the foot)

- Easy induction of the swing phase and adapted swing-phase control when using fitted parts for the knee

- Adapted foot properties (heel strike, heel-toe walking, solution for ball of foot; pro- and supination)

- Age-specific construction (considering stump condition)

- Restoration of external appearance. 


\section{Prothetik nach Amputationen im Bereich des Fußes}

Die Grundfrage einer jeden fußprothetischen Versorgung ist dahin gehend $\mathrm{zu}$ stellen, ob das obere und das untere Sprunggelenk unter den Gesichtspunkten der Gelenkfehlstellungen, der Gelenkbeweglichkeit oder der Kraftentfaltung noch erhalten und funktionell einsatzfähig sind.

Ist dieses der Fall, ist die Versorgung mit einer knöchelfreien Fußprothese anzustreben, da diese den Sprunggelenken ihre Beweglichkeit belässt. Damit verbleibt dem Amputierten die Möglichkeit, sein Gelenk aktiv einzusetzen, um sich z.B. am Ende der Standphase vom Boden abzustoßen oder sich auf unterschiedliche Absatzhöhen (in Grenzen) diverser Schuhe einzustellen.

Ist diese Eingangsfrage nur negativ zu beantworten, läuft die Verordnung auf eine sprunggelenkübergreifende Fußprothese inklusive der Einfassung des Unterschenkels hinaus, wobei der Patient in diesem Fall auf die biomechanischen Vorteile eines beweglichen Sprunggelenkes verzichten muss.

Abb. 1 zeigt die beiden grundlegenden Konstruktionen. Die Indikationen der knöchelfreien Fußprothese sind Amputationsniveaus im Bereich der Mittelfuß- und der Lisfranc-Stümpfe. Sprunggelenkübergreifende Konstruktionen sind notwendig bei Amputationsniveaus im Sprunggelenkbereich, z. B. bei Pirogow-Stümpfen mit den Modifikationen oder den Syme-Stümpfen.

Einen Sonderfall stellen in diesem $\mathrm{Zu}-$ sammenhang die Chopart-Stümpfe dar. Anatomisch betrachtet verfügen sie zwar über ein Sprunggelenk, welches in seinen funktionellen Möglichkeiten durch Fehlstellungen aber häufig so beeinträchtigt ist, dass eine Versorgung unter Einbeziehung des Unterschenkels notwendig ist. Bei Amputierten mit Chopart-Stümpfen ist deshalb immer eine Einzelfallentscheidung über das Versorgungskonzept nötig.

Es gibt eine Vielzahl von Konstruktionsbeispielen für Fußprothesen, die wichtigsten sind nachfolgend vorgestellt.

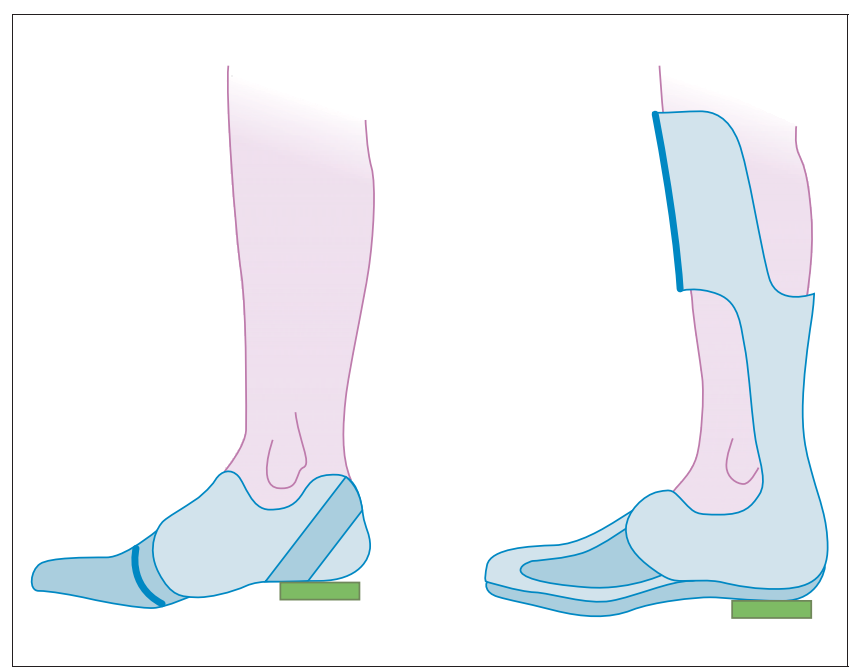

Abb. 1 Knöchelfreie und sprunggelenkübergreifende Fußprothese (BufaArchiv).

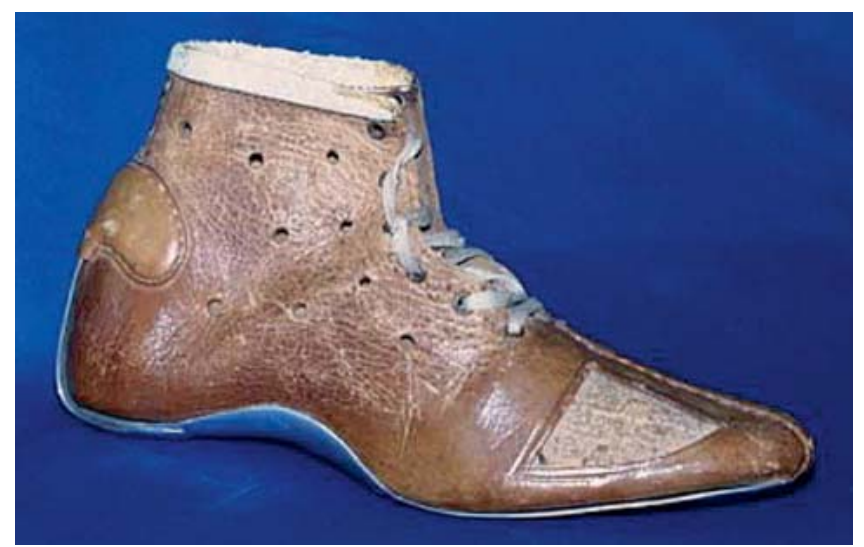

Abb. 2 Mobilisator nach Fendel (BufaArchiv).

\section{Knöchelfreie Fußprothesen}

\section{Mobilisator nach Fendel (Abb. 2)}

Hierbei handelt es sich um eine scheinbar überholte Prothesenkonstruktion, die aber Beachtung verdient, da sie erstmalig die biomechanischen Grundzüge knöchelfreier Fußprothesen zeigt. Somit ist der Mobilisator der Wegbereiter für moderne Konstruktionen, die das Sprunggelenk nicht übergreifen.

Die Prothesenfixierung ist hier verwirklicht durch eine Fersenklammer, die in die natürlichen Hinterschneidungen zwischen der Achillessehne und der Malleolen eingreift. Der erforderliche Haftmechanismus resultiert aus einer Ausnutzung der anatomischen Gegebenheiten, die einen großen Maßunterschied zwischen dem ML-Maß des Kalkaneus und dem ML-Maß des suprakalkanearen Bereiches aufweist. Die Prothese verklammert sich daher oberhalb des Kalkaneus.
Fußprothese nach Bellmann (Abb. 3)

Eine der aktuellen Weiterentwicklungen der Fendel'schen Konstruktion ist die knöchelfreie Fußprothese nach Bellmann. Auch sie haftet nach dem Prinzip der Fersenklammerung, jedoch in einer flexiblen Ausführung.

Schon bei der Herstellung des Gipsmodells muss die Absatzhöhe des Schuhes berücksichtigt werden, sodass dazu die Abrollung in der Prothese oder am Schuh eingebaut werden kann. Ein physiologischer Bewegungsablauf wird erreicht durch eine auf die dynamischen Ansprüche des Patienten abgestimmte plantare Karbonversteifung. Zur Erleichterung des Abrollvorganges beim Gehen kann gerade für Geriatriker der Abrollpunkt nach dorsal unter das Stumpfende verlagert sinnvoll sein. Diese Maßnahme verkürzt zwar minimal die Schrittlänge, kann aber für das effektivere Gehen hingenommen werden. 


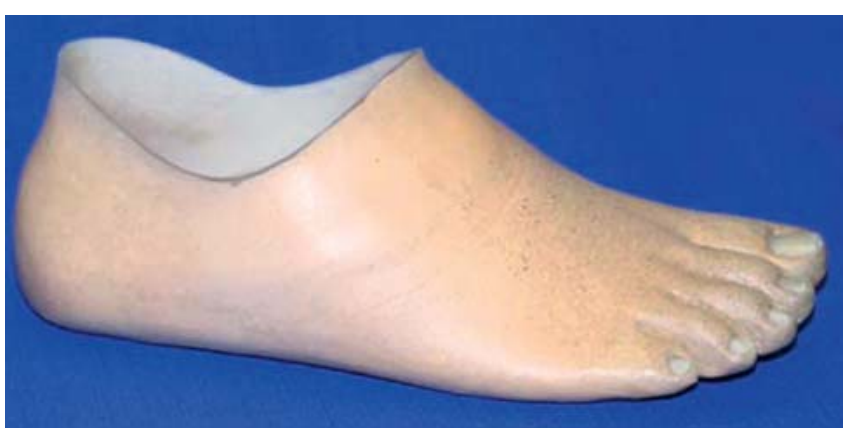

Abb. 3 Vorfußprothese nach Bellmann (Bufa-Archiv).

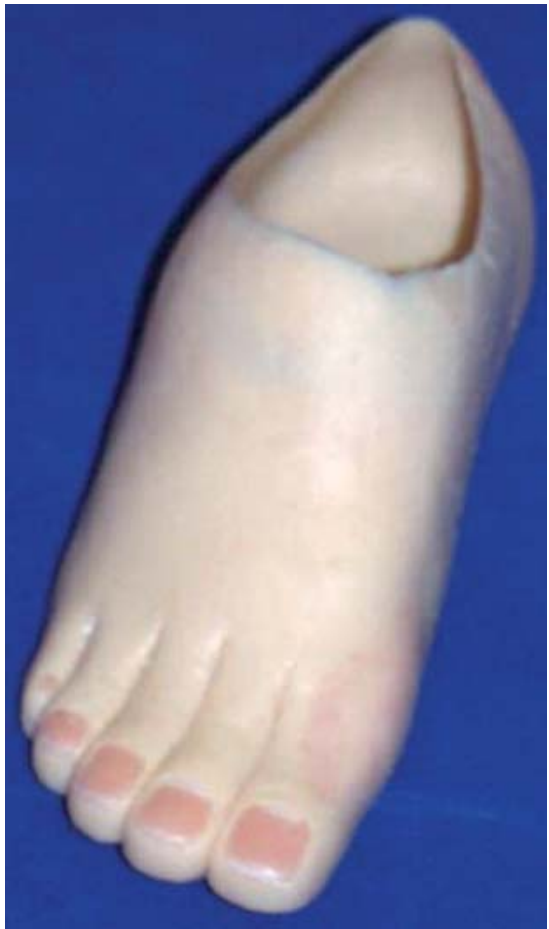

Abb. 4 Vorfußprothese aus Silikon (Bufa-Archiv).

\section{Vorfußprothese aus Silikon (Abb. 4)}

Sie unterscheidet sich von anderen knöchelfreien Fußprothesen insbesondere durch einen anderen Haftmechanismus. Eine auf der anatomischen Topografie beruhende Fersenklammer wird nicht benötigt. Die Prothese haftet am Stumpf aufgrund des hohen Haftreibungskoeffizienten zwischen Haut und Silikon.

In Bezug auf die kosmetische Ausgestaltung ermöglicht eine Silikonvorfußprothese derzeit die größtmögliche Annäherung an das natürliche Vorbild, ein Nachteil ist das relativ hohe Eigengewicht solcher Konstruktionen.

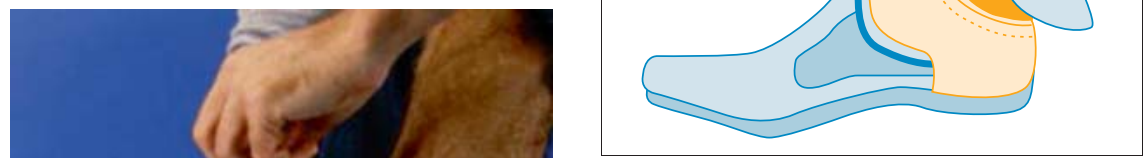

Abb. 6 Rückfußprothese mit Einstiegsklappe (Bufa-Archiv).

\section{Rückfußprothese mit Einstiegsklappe}

(Abb. 6)

Eine Möglichkeit der Fußprothese mit Unterschenkelfassung ist die Ausführung mit Einstiegsklappe. Sie ermöglicht, dass auch kolbige Stumpfenden problemlos in die Prothese eingeführt werden können. Über einen Führungsstift und einen geeigneten Verschluss wird die Einstiegsklappe mit der Prothese verbunden und somit die Prothese am Körper des Patienten fixiert.

Abb. 5 Rahmenprothese nach Botta (Fa. Botta, CH).

\section{Rückfußprothese mit Innenschaft} (Abb. 7)

\section{Sprunggelenkübergreifende Fußprothesen}

\section{Rahmenprothese (Abb.5)}

Bei den Fußwurzelstümpfen ist die Beweglichkeit des oberen Sprunggelenkes zum kraftvollen Abdrücken am Ende der Standphase nicht mehr voll vorhanden. Der fehlende Vorfußhebel kann nur durch eine nach proximal hoch gezogene Ristlasche kompensiert werden. Wenn das obere Sprunggelenk noch einige Winkelgrade an Beweglichkeit zulässt, so kann diese durch eine Rahmenkonstruktion nach Botta ausgenutzt werden.

Diese Rückfußprothese in Rahmenkonstruktion ist besonders für muskelkräftige Patienten geeignet. Der Rahmen des Prothesenschaftes muss so gestaltet werden, dass der Amputierte das kolbige Stumpfende durch die Freiräume in den Schaft „einfädeln“ kann.

Der Syme-Stumpf, dessen Amputationsart in Deutschland nicht sehr verbreitet ist, hat ein weniger kolbiges Aussehen. Er kann mit einem Weichwandinnenschaft aus einem Polyethylen-Schaummaterial ausgestattet werden, wie es auch bei den Unterschenkelprothesen üblich ist. Dieser vom Amputierten zuerst angezogene Innenschaft gleicht die größten Hinterschneidungen des Stumpfes aus, sodass ein leichtes $\mathrm{Hi}$ neingleiten mit dem nun konischen $\mathrm{Ge}$ gebenheiten in den harten, tragenden und mit dem Fuß verbundenen Außencontainer ermöglicht wird.

Lediglich bei der Syme-Amputation (evtl. auch bei den Pirogow-Modifikationen) ist es möglich, ein vorgefertigtes Fußpassteil mit einer geringen Bauhöhe zu verwenden. Bei allen anderen Versorgungen des Fußes müssen die Sohle, der Vorfuß und die Zehen individuell handwerklich hergestellt werden. 


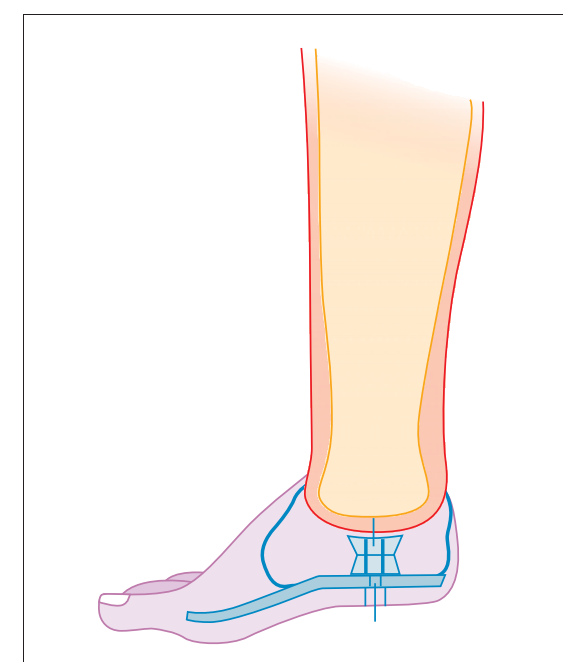

Abb. 7 Rückfußprothese mit Innenschaft (Bufa-Archiv).

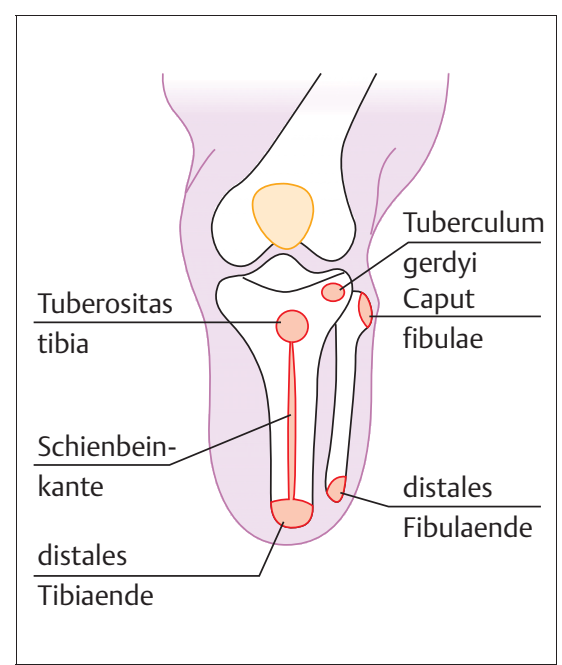

Abb. 8 Entlastungszonen am Unterschenkel (Bufa-Archiv).

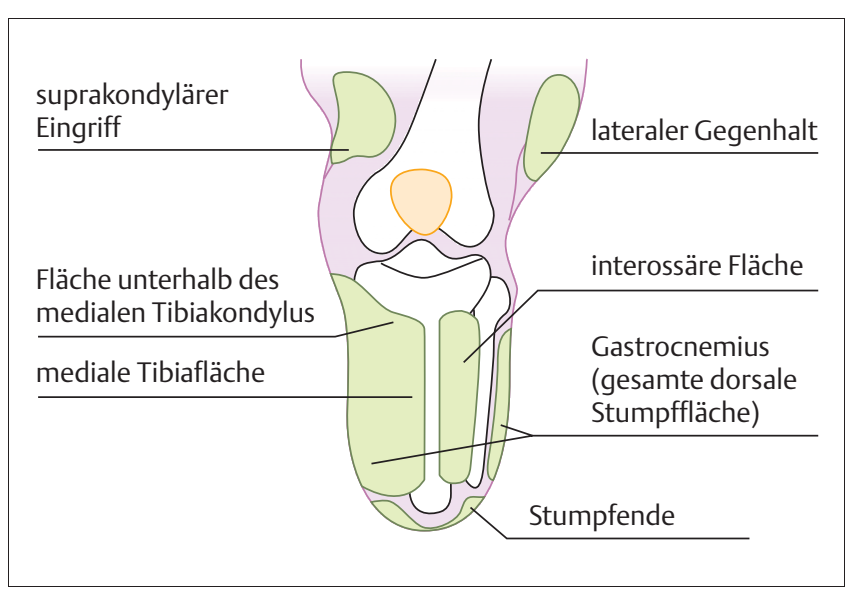

\section{Prothetik nach transtibialer Amputation}

Eine exakte Aufnahme des Stumpfvolumens hat unbedingte Priorität in der Gestaltung des Schaftes einer Unterschenkelprothese. Dabei müssen folgende Grundanforderungen erfüllt werden:

- Der Stumpf muss vollflächig anliegen

- Endkontakt muss vorhanden sein (auch und gerade bei Gefäßpatienten)

- Der Stumpf muss unter Berücksichtigung empfindlicher Knochenprominenzen eingepasst sein.

\section{Prinzip der Lastübertragung durch Zweckformung}

Grundsätzliches Ziel der Stumpfbettung ist die gleichmäßige Lastverteilung auf die gesamte Stumpfoberfläche. Für den
Abb. 9 Belastungszonen am Unterschenkel (BufaArchiv).

Amputierten wird der Druck verträglich, wenn die Kontaktfläche größtmöglich ist und die punktuelle Belastung so gering wie möglich.

Beschriebener Ansatz geht von der Tatsache aus, dass ein Unterschenkelstumpf über einen relativ großen Knochenanteil und eine vergleichsweise geringe Weichteildeckung verfügt. Diese Tatsache bedingt eine große Anzahl von Knochenprominenzen direkt unter der Hautoberfläche, die potenziell als Druckstellen in Erscheinung treten können. Aus diesem Grund wird die Stumpfoberfläche in belastungsfähige (Abb. 8) und druckempfindliche Areale (Abb.9) eingeteilt, die in der Modelltechnik getrennt berücksichtigt werden müssen, um eine optimale Druckverteilung über die gesamte Stumpfoberfläche zu erreichen.

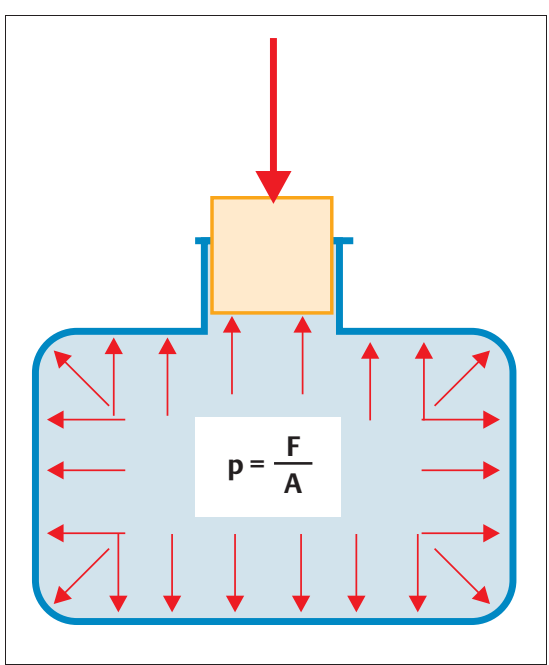

Abb.10 Verteilung von Kräften im hydrostatischen System (Bufa-Archiv).

\section{Prinzip der Lastübertragung durch Hydrostatik}

Bei diesem System wird davon ausgegangen, dass sich Flüssigkeiten - bzw. auch Weichteile des menschlichen Körpers - dazu eignen, Kräfte zu übertragen. Die Voraussetzung dafür ist gegeben, wenn sich die Flüssigkeit (Stumpf) in einem abgeschlossenen Behälter (Schaft) befindet und somit nicht entweichen kann. Aufgebrachter Druck verteilt sich gleichmäßig in dem gesamten Medium. Da sich Flüssigkeiten nicht komprimieren lassen, bilden sie unter diesen Bedingungen ein stabiles Gebilde; und es ist möglich auf diese Art und Weise Kräfte zu übertragen (Abb.10).

Vorteil der hydrostatischen Lastübertragung in einer Prothese ist der Effekt einer Minimierung des Drucks an jedem Punkt der Stumpfoberfläche durch Maximierung der Kontaktfläche zwischen Stumpf und Schaft nach dem bekannten physikalischen Gesetz.

Trotz der optimal gleichmäßigen Druckverteilung an der Stumpfoberfläche bleibt auch unter den Bedingungen einer hydrostatischen Lastübertragung das Problem einer Verlagerung der Knochen im Weichteilmantel nach wie vor erhalten, sodass Knochenprominenzen noch immer einer Druckproblematik ausgesetzt sind und entsprechend entlastet werden müssen. 


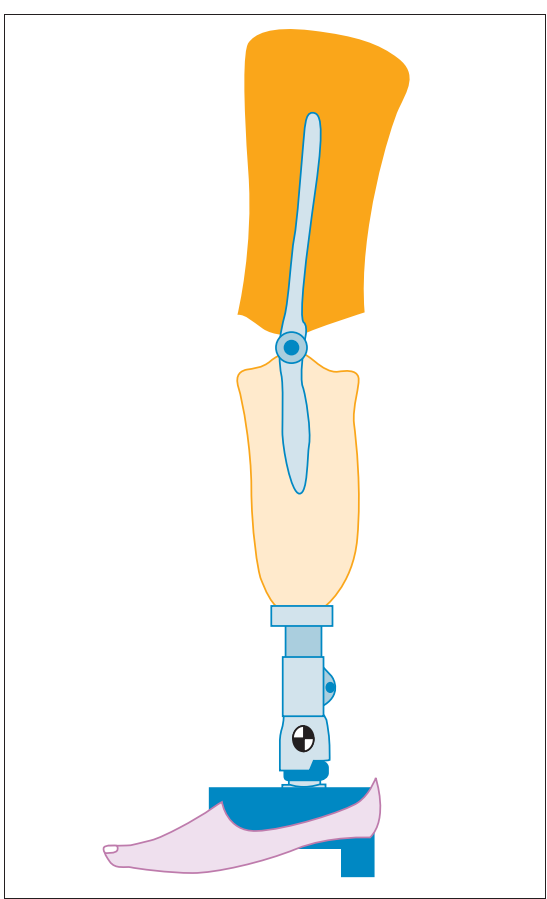

Abb. 11 Unterschenkelprothese mit Oberhülse (Bufa-Archiv).

\section{Unterschenkelprothesen mit Oberhülse} (Abb.11)

Bei der Versorgung eines Patienten mit einer Unterschenkelprothese mit Oberhülse kommen folgende Merkmale zum Tragen: Die Hauptkörperlast wird über die Verschnürung der Oberschenkelmanschette aufgenommen. Das kann im nachteiligen Sinne zur Atrophie der Oberschenkelmuskulatur führen und $\mathrm{zu}$ Stauungsproblemen der Blutzirkulation. Das Kniegelenk betreffend kann gesagt werden, dass die Kongruenz zwischen Schienengelenk- und Kompromissdrehpunkt nur selten und dann zufällig beim täglichen Anlegen vom Patienten erhalten bleibt. Die Folge dieser Inkongruenz ist eine unphysiologische Belastung des Gelenkes mit der Gefahr einer verfrühten Arthrose.

Im vorteiligen Sinne können so Patienten versorgt werden, deren Stumpf nur unzureichend belastbar ist, vielleicht aufgrund einer Entzündung, das Vorhandensein eines Neuroms oder einer Unbelastbarkeit der Hautoberfläche (z. B. durch Kelloide, Spalthaut, Meshgraft).

Aufgrund beschriebener Nachteile wird diese Form der Prothesenkonstruktion nur noch selten eingesetzt.

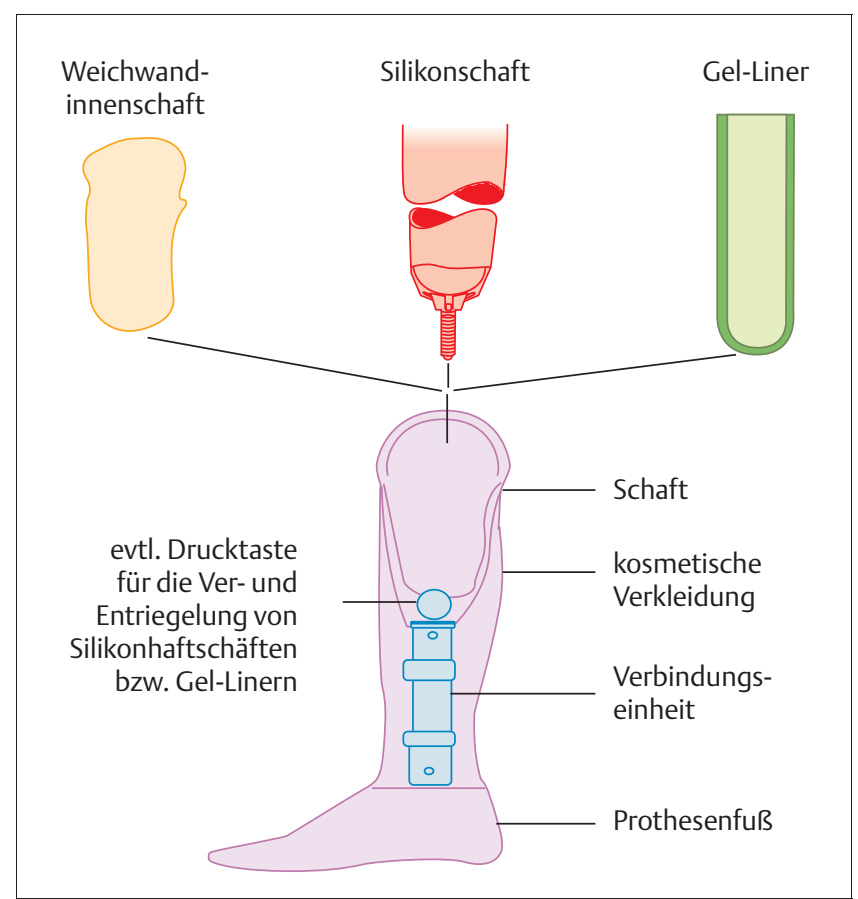

Abb. 12 Diverse Haftungsmöglichkeiten einer Unterschenkelprothese (Bufa-Archiv).
Unterschenkelprothese mit suprakondylärer Fassung (Abb.12)

Bereits im Jahr 1925 hat Görlach eine manschettenlose Unterschenkelprothese vorgestellt, die nur noch durch eine schmale zirkuläre Kniebandage gehalten wurde. Aber erst Radcliffe und Foort haben durch ihre biomechanischen Grundlagen 1958 die Entwicklung der PTB-Prothese (Patella Tendon Bearing) ermöglicht. Der hierbei exakt modellierte und unter biomechanischen Gesichtspunkten gestaltete Schaft machte es möglich, dass der Stumpf tatsächlich das gesamte Körpergewicht tragen konnte. Die Schaftaufgaben einer vollflächigen Gewichts- und einer spielfreien Bewegungsübertragung waren damit erfüllt. Nur für die Haftung der PTB-Prothese musste nach wie vor eine schmale Haltebandage verwendet werden.

Gerade aber die strangulierende Wirkung dieser Kniebandage veranlasste weitere Entwicklungen. 1960 stellte Fajal die P.T.S. (Prothèses Tibiale Supracondylienne) vor, bei der die Kniescheibe vollkommen im Schaft eingebettet war und so ein Abrutschen der Prothese verhinderte.

1963 folgte Kuhn mit der KBM-Prothese (Kondylen-Bettung Münster). In dieser Konstruktion wird der Kontakt zwischen Stumpf und Prothesenschaft durch die Verklammerung des medialen Randes oberhalb der Kondyle erreicht. Zum leichteren Anziehen konnte der sehr enge Kondylenbereich durch Entfernen der medialen Backe („Kondylenkeil“) erweitert werden, sodass der Patient mit seinem Knie die Engstelle passieren und seinen Stumpf in den Schaft einführen konnte. Nach erneutem platzieren des Kondylenkeiles wurde der Schaft eng mit dem Stumpf verbunden.

Basierend auf den Prinzipien der KBMProthese kommen die Weiterentwicklungen aufgrund der Gestaltung des Weichwandschaftes (Soft-Socket) und der eingesetzten Armierungsmaterialien für den harten Außencontainer ohne separaten Kondylenkeil aus.

\section{Unterschenkelprothese mit Silikon- haftschaft (Abb. 12)}

Im Bereich der Lastübertragung folgt dieser Prothesentyp selbstverständlich auch den oben beschriebenen biomechanischen Gesetzmäßigkeiten. Hierin unterscheidet er sich nicht von der Prothese mit suprakondylärer Fassung und kann somit auch in der Formgestaltung analog dazu betrachtet werden. Im Gegensatz dazu aber beruht die Fixierung des Silikonhaftschaftes auf einem völlig anderen Mechanismus. Zur Anwendung kommt hier die hohe Haftreibung des Silikonmaterials auf der Hautoberflä- 
che. Diese bewirkt eine unverrückbare Adaption des Silikonmaterials auf dem Stumpf. Die Verbindung zum festen Prothesenschaft wird durch ein zusätzliches mechanisches Verschlussstück (shuttle-lock, clutch-lock etc.) oder durch eine Dichtlippe erreicht. Der Vorteil eines Silikonhaftschaftes liegt darin, dass dieser nach dem heutigen Stand der Technik die innigste Verbindung zwischen Stumpf und Prothese bewirkt. Ein prinzipieller Nachteil liegt in der Gefahr, dass der dünnwandige und hochelastische Silikonmantel beim Durchschwingen der Prothese Zugbelastungen auf das Stumpfende des Amputierten ausübt. Diesem Problem kann z. B. durch gezielte Verstärkungen (zur Einschränkung der Längszügigkeit) im Silikonmaterial begegnet werden. Für die Mehrzahl der Unterschenkelamputationen steht ein ausreichendes Sortiment an industriell vorgefertigten Silikonhaftschäften zur Verfügung. Im Einzelfall (z. B. ungewöhnliche Stumpfformen) können individuelle Anfertigungen notwendig sein.

In Bezug auf den stabilen, lastübertragenden Prothesenschaft erfolgt bei Versorgungen mit Silikonhaftschaftprothesen zunächst obligatorisch die Anfertigung eines klarsichtigen Testschaftes, der anschließend nach den Anproben durch einen hochbelastbaren Definitivschaft aus Faserverbundwerkstoff ersetzt wird.

\section{Unterschenkelprothese mit „Gel-Innenschaft““(Abb.12)}

Das grundsätzliche Ziel bei der Verwendung von Gel-Innenschäften in Bezug auf die Übertragung von Kräften zwischen Stumpf und Schaft ist wiederum die Nutzung einer möglichst großen Lastübertragungsfläche, um somit eine gleichmäßige Druckverteilung und einen geringen Flächendruck an der gesamten Stumpfoberfläche zu erreichen. Die Lösung dieses Grundproblems unterscheidet sich von Prothesentyp zu Prothesentyp. Das spezifische an USProthesen mit Gel-Innenschaft ist, dass hier die typischen Materialeigenschaften der verwendeten Gel-Produkte zur Wirkung kommen. Diese sind:
- Hohe Verdrängungsmöglichkeit des Materials: Hierdurch wird eine Umverteilung des Gel-Materials von empfindlichen Knochenprominenzen zu den angrenzenden Arealen bewirkt, sodass es zu einem Ausgleich der Druckbelastung am Stumpf kommt.

- Optimales Memory-Verhalten des Materials: Da die Belastungssituation des Stumpfes im Schaft keine statische ist, sondern sich dynamisch ändert, muss der oben beschriebene Prozess reversibel sein, sodass er von Lastwechsel zu Lastwechsel neu starten und zu einer permanenten Anpassung an wechselnde Druckverhältnisse führen kann. Dies wird durch das Memory-Verhalten des Materials ermöglicht.

Für die Haftung von Gel-Liner-Prothesen an der Extremität gibt es eine produktspezifisch breite Streuung. Die Haftmechanismen reichen von einer suprakondylären Fassung über Haftreibungseigenschaften in Verbindung mit mechanischen Verschlusssystemen bis hin zur Haftung über Druckdifferenz, wobei über ein Ventil die Luft aus dem Prothesenschaft verdrängt wird und ein luftdichter Verschluss ein zurückströmen der Luft verhindert.

Auch bei Gel-Innenschäften gibt es die Wahl zwischen vorkonfektionierten und individuell angefertigten Produkten.

Die Anfertigung des stabilen, lastübertragenden Prothesenschaftes erfolgt in gleicher Weise wie bei Silikonhaftschaftprothesen.

\section{Prothetik nach Knieexartikulation}

Diese Form der Absetzung des Unterschenkels erzwingt den Einsatz eines künstlichen Kniegelenkes, sodass hier zwischen dem Schaft, dem Kniegelenkund dem Fußpassteil unterschieden werden muss.

Die Knieexartikulation hat eine ganze Reihe biomechanischer Vorteile gegenüber den Amputationen im Bereich des Röhrenknochens (Femur):

- Die gute Belastbarkeit der breiten Kondylenflächen ist ein entscheidender Punkt für die Übertragung des Körpergewichtes

- Durch die Stumpfendbelastung entsteht ein Bodengefühl, das den Patienten ein sicheres Standgefühl vermittelt
- Das Anziehen und Ablegen der Prothese ist ohne nennenswerte Probleme auch für die geriatrischen Patienten leicht durchzuführen, da in der sitzenden Position keine unerwünschten Verdrehungen der Prothese möglich sind

- Der Stumpf als Hebelarm ist lang und die Steuerung der Prothese ist deshalb vorteilhafter als bei einem Oberschenkelstumpf

- Die Breite der Kniekondylen dient dazu, den Prothesenschaft am Stumpf zu fixieren, diese sollten deshalb nicht operativ verschmälert werden.

Der größte Nachteil der Knieexartikulation ist die kosmetisch ungünstige Länge des Amputationsstumpfes, die durch das Prothesenkniegelenk noch verstärkt wird. Die Entwicklung entsprechender Kniegelenke ist die konstruktive Antwort auf diese Problemstellung, sodass praktisch nur Prothesen in der Modulartechnik befriedigende Ergebnisse zulassen. Leider ist auch in den so wenig ansprechenden früheren prothetischen Versorgungen eine der Ursachen dafür zu erkennen, dass die Vorteile dieser Art der Amputation lange Zeit nicht erkannt wurden.

Auch bei der Versorgung nach Knieexartikulation muss der Schaft individuell auf einem Gipsmodell hergestellt werden. Dabei hat die Modellierung der Kniekondylen mit besonderer Sorgfalt zu erfolgen, da diese einerseits das Körpergewicht tragen sollen und andererseits die Haut dieser Stumpfzone nicht oder nur geringfügig mit Weichteilen gepolstert ist. Es ist absolut notwendig, dass dem Stumpf weder in vertikaler noch in horizontaler Richtung pseudarthrotische Bewegungsmöglichkeiten ermöglicht werden.

Die biomechanischen Aufgaben des Schaftes sehen wie folgt aus:

- Gewichtsübertragung durch den distalen Stumpfbereich, aber auch über die gesamte Stumpfoberfläche

- Die Bewegungsübertragung wird durch eine formschlüssige Schaftgestaltung im Kondylenbereich gewährleistet

- Die Haftung des Schaftes am Stumpf wird durch eine Verklammerung oberhalb der Epikondylen erreicht, ähnlich der Unterschenkelkurzprothese. 


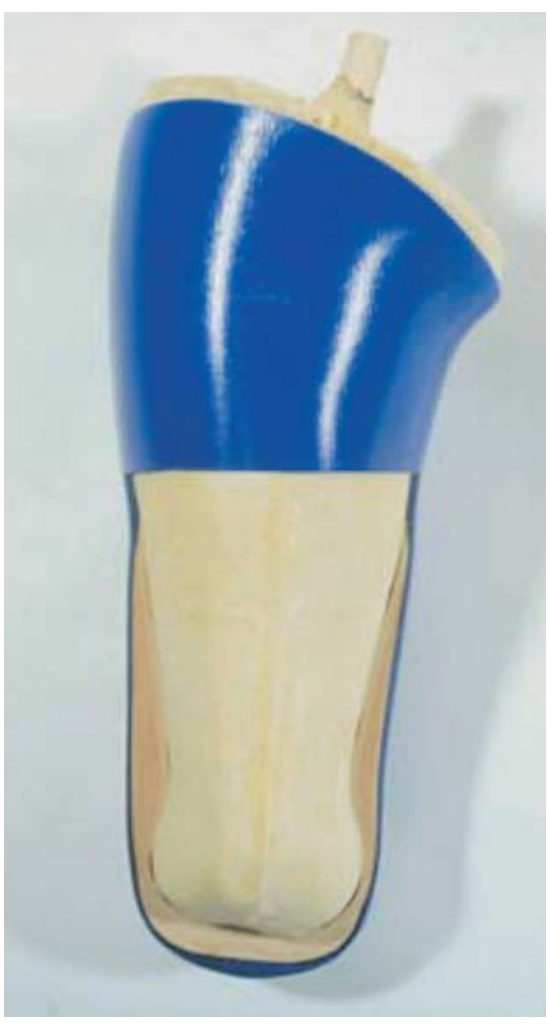

Abb. 13 Stumpffassung einer Knieexartikulationsprothese (Bufa-Archiv).

Um diese Aufgaben ohne Hautschädigung zu erreichen, besteht auch der Knieexartikulationsschaft aus zwei Teilen. Der weiche Innenschaft aus einem Polyethylen-Schaum (Soft-Socket) gleicht die Hinterschneidungen des Stumpfes aus und gestaltet diesen so zu einem Konus oder Zylinder, mit dem dann ein leichteres Hineingleiten in den Außencontainer ermöglicht wird (Abb.13). Beide Schaftteile reichen medial bis unterhalb des Perineums, lateral bis auf die Höhe des Trochanter majors.

Der Außencontainer ist nach distal rigide und formstabil armiert, damit die Befestigungsteile des Kniegelenkes angebracht werden können. Der proximale Schaftrand wird im Allgemeinen flexibel gestaltet. Der so entstandene verformbare Rand passt sich beim Sitzen der Unterlage an und hat noch genügend Festigkeit, um in der Standphase ausreichend Stabilität zu geben. Durch den Anpressdruck des Stumpfes können sich die Schaftteile nicht von allein lösen. Bei sehr kolbigen Stümpfen kann der SoftSocket mit einer Lasche versehen werden, die den Schaft beim Anziehen weiten lässt und das Anziehen erleichtert.

\section{Prothetik nach transfemoraler Amputation}

Grundlagenforschungen zur Biomechanik einer Oberschenkelprothese haben deutlich gemacht, dass es nur Grundformen in der Schaftgestaltung gibt, die an die individuellen Körperdimensionen angepasst werden müssen. Diese Anpassung ist notwendig, weil alle statischen und kinematischen Kräfte in unphysiologischer Weise vom Körper über einen respektablen Weichteilmantel des Oberschenkels auf die Prothese übertragen werden müssen. Schwierigkeiten bietet die Anpassung auch aufgrund des Mangels an objektiven Parametern zur Bestimmung des Verhältnisses von Stumpf- und Schaftvolumen. Wie schon bei der transtibialen Prothese beschrieben, gehört auch nach einer transfemoralen Amputation die vollständige Aufnahme des Stumpfvolumens zu den wichtigsten Aufgaben des Schaftes. Nur mit der Gewährleistung dieser Forderung sind die weiteren Aufgaben, Haftung zu erzielen und die auftretenden Kräfte in vertikaler und horizontaler Richtung zu übertragen, abzuleiten.

Nachfolgend werden die Haftmechanismen einer Oberschenkelprothese dargestellt. Der hydrostatische Druck ist die flüssigkeitsbedingte Druckweiterleitung nach dem Pascal'schen Gesetz, wenn man vereinfacht die festeren Strukturen des menschlichen Gewebes vernachlässigt. Im Oberschenkel-Prothesenschaft müssen die Weichteile unter einer gewissen Kompression stehen, sonst wäre eine Steuerung der Prothese in der Schwungphase nicht erreichbar und in der Standphase die Übertragung des Körpergewichtes nicht möglich. Der Schaft hat darum auch ein kleineres Volumen als der Stumpf.

Der elastische Seitendruck entsteht durch das Einziehen des Stumpfes z.B. mit einem Trikot in den Schaft. Der Anziehtrikot wird völlig aus dem Schaft herausgezogen. Dabei werden die oberflächlichen Strukturen der Haut etwas tiefer in den Schaft gezogen. Diese Hautvorspannung reduziert die StumpfSchaft-Pseudarthrose.

Der muskuläre Druck ist im Prinzip die Folge der hydrostatischen Druckwirkung und der Schaftform. Er bewirkt bei Kontraktion der Muskulatur eine Verspreizung in der Schaftform und eine partielle Verstärkung der Pressanlage. Diese muskuläre Verspreizung ist während des gesamten Doppelschrittes in unterschiedlichen Stumpfbereichen wirksam.

Die Saugwirkung: Da durch das Muskelspiel und der geringen Dauerdruckbelastung der Haut $(<2,5 \mathrm{~N} / \mathrm{cm})$ kein extrem enger Schaft gestaltet werden kann, ist nicht sichergestellt, dass der Schaft nicht doch wieder vom Stumpf abrutscht. Der letzte und sicherste Haftfaktor ist die Saugwirkung, die durch den Verschluss des Einziehloches mit einem Gummiflachventil erzielt wird. Dieses Ventil lässt nur Luft in den und aus dem Schaft, wenn ein kleiner Metallknopf gedrückt wird. Aufgrund der anderen wirksamen Haftmechanismen sollte nur von einem Saugschaft gesprochen werden, wenn ein automatisches Ausstoßventil benutzt wird. In der Praxis zeigt sich aber immer wieder eine Blauverfärbung am Stumpfende. Aufgrund der sehr starken Saugwirkung kommt es zur Abflussbehinderung des Blutes. Ausstoßventile werden deshalb nicht mehr verwendet.

Die Haftreibung entsteht beim Kontakt verschiedener Stoffe aufeinander (Adhäsion). Bei der Verwendung unterschiedlicher Materialien für die Oberschenkelschäfte wird deutlich, dass ein wesentlicher Faktor der Haftung auch in der Materialkombination liegt und damit eine technische Kenngröße, die Haftreibzahl $\mu_{0}$, von Bedeutung ist. Gerade bei kurzen Stümpfen, die eine kleine Oberfläche besitzen, ist dieser Reibungskoeffizient besonders wichtig.

\section{Schaftvariationen}

Wohl aufgrund der allgemeinen Schwierigkeiten, die ein Oberschenkelamputierter mit der Prothese haben kann, gibt es eine Vielzahl von Schaftvarianten.

\section{Interimschaft}

Gerade nach transfemoraler Amputation machen sich Volumensschwankungen auf die Steuerbarkeit der Prothese sofort bemerkbar. Oberschenkelamputierte Patienten benötigen im Verhältnis zu distaleren Amputationen auch eindeutig mehr Zeit und mehr Training, bis sie die Prothese beherrschen. Der Interimschaft bietet die Möglichkeit, sehr schnell einen biomechanisch korrekten und doch leicht veränderbaren Prothesenschaft zur Frühmobilisierung zu schaffen. Das Knie- und Fußpassteil kann bereits definitiv bestimmt sein 


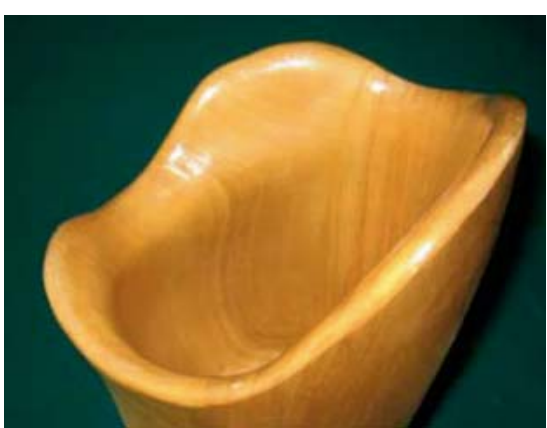

Abb. 14 Sitzbeinumgreifender Oberschenkelschaft aus Holz (Bufa-Archiv).

mit der Option des leicht auszuführenden Gelenkwechsels bei Mobilitätssteigerung. Die Interimprothese ist also keine minderwertige, sondern eine nur temporär vorgesehene Versorgung.

\section{Holzschaft (Abb.14)}

Der Holzschaft ist zumindest in Deutschland nach dem 2. Weltkrieg zur Standardversorgung geworden. Alle Arbeitstechniken wie Aufbau- und Justiermethoden sowie Werkzeuge und Maschinen waren auf dieses Material ausgerichtet. Die Vorteile dieses Werkstoffes sind in der relativ leichten Verarbeitung und Veränderbarkeit des Materials zu finden. Die Indikation liegt bei Patienten mit Neigung zu allergischen Reaktionen auf Kunststoffe; für diese darf der Innenraum des Stumpfbettes im Holzschaft dann auch schon einmal unlackiert bleiben oder nur gewachst werden. Durch modernere Techniken und dem Einsatz von Kunststoffen ist die Verwendung von Holz aber stark rückläufig.

\section{Gießharzschaft}

Der Gießharzschaft besteht aus dem Faserverbundwerkstoff Acrylharz, sodass dieser dünnwandig, aber sehr stabil ist. Durch die Wasserfestigkeit des Materials konnten nun auch Prothesen zum Baden und Duschen hergestellt werden. Die weitere Indikationstellung für den Gießharzschaft sind Patienten, die eine sehr starke Schweißabsonderung aufweisen.

\section{Flexibler Innenschaft mit Spangen- container}

Der flexible Innenschaft als neuere Prothesentechnik ist seit 1983 bekannt. Entwickelt wurde er in Island aufgrund der großen Zahl von zu versorgenden

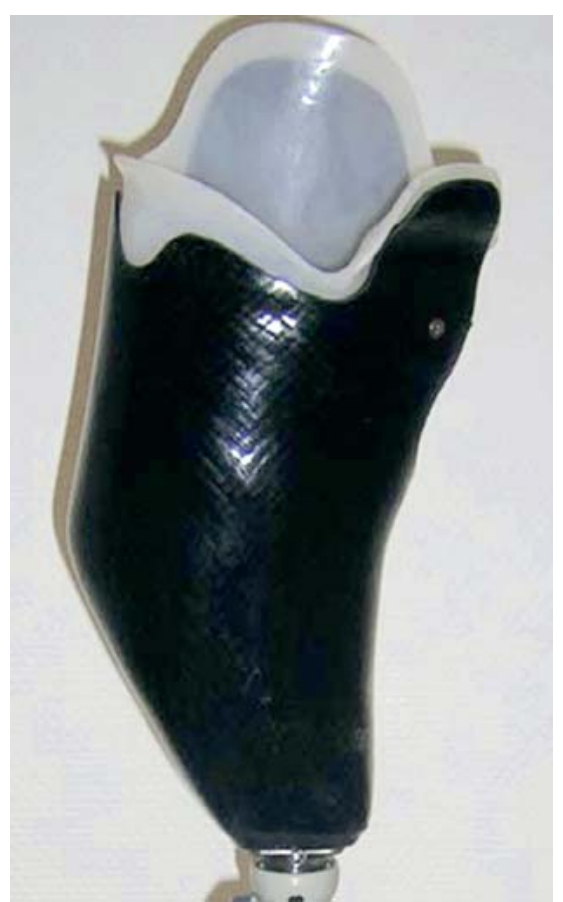

Abb. 15 Sitzbeinumgreifende Oberschenkelprothese mit flexiblem Innenschaft und rigidem Außencontainer (Bufa-Archiv).

Geriatrikern. Es zeigte sich aber schnell, dass nicht nur diese Patientengruppe von diesem Schaftsystem profitierte. Allgemein bekannt ist er nun unter dem Kürzel ISNY-Schaft. Der originale ISNYSchaft besteht aus einem sehr dünnen und dadurch flexiblen Polyethylen und aus einem tragenden, mit Carbonfasern armierten Außenschaft, der nur spangenartig die wichtigsten Belastungspunkte unterstützt. Auf diese Weise sind $3 / 4$ der Stumpfoberfläche nur mit dem flexiblen Material bedeckt.

Die aufwendigere Herstellung dieses Schaftes ist durch die Vorteile gerechtfertigt:

- Verbesserter Tragekomfort beim Gehen und Sitzen

- Geringes Gewicht

- Sensible Rückmeldung

- Gutes Wärmeverhalten

- Spielraum für Muskelaktivität

- Gute Haftung.

\section{Flexibler Innenschaft mit rigidem Außencontainer}

Dieser den Innenschaft vollständig umschließende Container ist eine Variation des Spangenschaftes; unabhängig von der Formgestaltung. Er besteht aus zwei leicht voneinander zu trennenden Anteilen, und zwar dem flexiblen (Innen-)Schaft und einer äußeren tragen- den Hülle aus einem Carbonfaser-Laminat (Abb.15). Der Raum zwischen dem Stumpfende des Innenschaftes und dem Kniegelenksadapter im Außenschaft kann frei gefertigt werden. Damit wird das Gesamtgewicht reduziert und es ist möglich, Veränderungen im distalen Schaftbereich durchzuführen.

Beide Systeme sind indiziert für Geriatriker, für Gefäßpatienten, bei muskelkräftigen Amputierten oder auch bei kurzen Stümpfen; vor allem das Containersystem gilt heute als Standardversorgung.

\section{Die biomechanische Formgestaltung des Oberschenkelschaftes}

Seit jeher wird über die „richtige“ Schaftform gestritten, selbst nachdem sich biomechanische Erkenntnisse durchgesetzt haben. Gerade dieses Wissen lässt nach neuen Wegen suchen, und deshalb wird die Diskussion auch nicht abbrechen. Bevor die Formkriterien und die daraus resultierenden Schaftformen genauer dargestellt werden, müssen noch einige Begriffe genannt sein.

Nach Hepp und Elle (1948) kann ein Oberschenkelschaft in drei Ebenen mit unterschiedlichen Funktionen eingeteilt werden:

- I. Ebene: Sitzringbereich; vom proximalen Schaftrand bis ca. $5 \mathrm{~cm}$ nach distal

- II. Ebene: Steuerungsbereich; erstreckt sich nach distal bis zum Femurende

- III. Ebene: Stumpfendbereich; umfasst die Stumpfkuppe mit dem Einziehloch.

\section{Die Zweckformung der Stumpf-} eintrittsebene

Die Stumpfeintrittsebene für einen Schaft nach transfemoraler Amputation ist heute in zwei Grundformen einteilbar. Abb.16 zeigt die Grundformen von zwei Oberschenkelschäften als Querschnitte im Verhältnis zu den Knochenund Muskelstrukturen des Beckens.

Auf der linken Seite ist ein querovaler Schaft, der auch als sitzbeinunterstützender Schaft bezeichnet wird, abgebildet. Im Gegensatz dazu ist der rechtsseitige Schaft in der Längsrichtung oval und kann deshalb als längsovaler Schaft oder als sitzbeinumgreifender Schaft bezeichnet werden. 


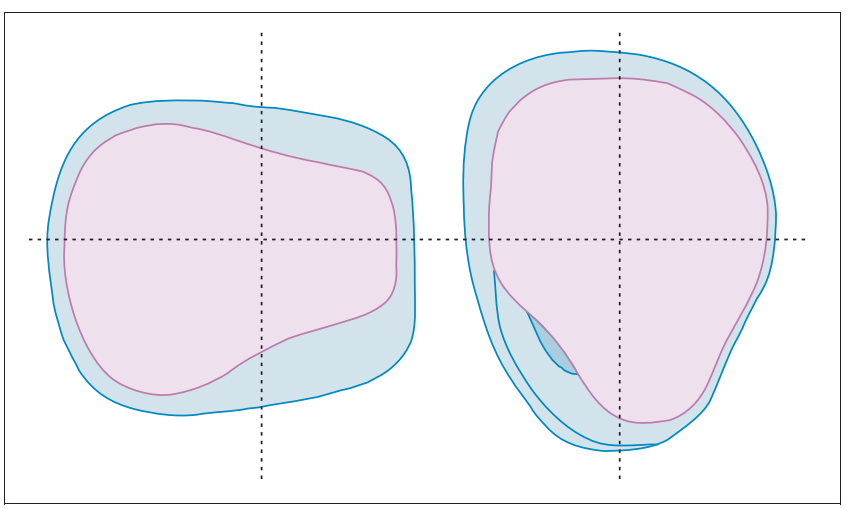

Abb. 16 Querschnitt des sitzbeinunterstützenden (links) und sitzbeinumgreifenden Schaftes (rechts) (BufaArchiv).
Querovale Schäfte versuchen durch die Zweckformung ihres proximalen Querschnittes das Tuber ischiadicum auf dem vorgesehenen Stützbereich am Prothesenschaft zu halten, um das Körpergewicht auf die Prothese übertragen zu können. Deshalb ist es notwendig, insbesondere den $M$. semimembranosus, den M. semitendinosus und den M. biceps femoris aus ihrer natürlichen Lage nach vorn zu verdrängen. Sie müssen sich um den dorsalen Schaftrand legen, damit das Sitzbein auf dem Rand aufsitzen kann. Diese Verdrängung funktioniert nur, wenn es einen entsprechenden Gegenhalt im frontalen Bereich des Schaftes gibt; die sog. Frontalpelotte. Sitzbeinunterstützende Schäfte müssen deshalb ein relativ schmales Perineum aufweisen. Für das Perineum wird ein durchschnittlicher Abstand der Adduktorensehne bis zum Tuber ischiadicum von 7-11 cm zugrunde gelegt.

Da dieser Kontaktpunkt Tuber/Schaftrand hinter und die Schwerkraft vor dem Drehpunkt des Hüftgelenkes wirkt, entsteht in der Sagittalebene unweigerlich ein beckenvorkippendes Drehmoment (Abb. 17). Die gegenüber dem Tuberaufsitz liegende Frontalpelotte fängt dieses Drehmoment auf und hält das Sitzbein auf der sog. Tuberbank. Leider befindet sich diese Pelotte in einem Bereich, in dem die Femoralgefäße (Arteria und Vena femoralis, Nervus femoralis) oberflächlich liegen und somit nicht gegen Druck geschützt sind. Gerade bei durchblutungsgestörten Amputierten entstehen so massive Probleme. Aber auch jüngere und hier gerade die sportlich aktiven Patienten empfinden den permanenten Druck am Sitzbein als unangenehm bzw. hinderlich. Bei diesen Patienten wird die Tuberbank meist sehr abgerundet und flach verlaufend gestaltet sein müssen.
Die Hinsicht auf das heutige und vor allem in der Zukunft zu erwartende Patientenklientel (pAVK-Patienten, Diabetes als besondere Form der AVK usw.) lässt erkennen, dass diese Schaftform mit ihren Eigenschaften nicht die gewünschte sein kann.

Die immer häufiger gestellte Forderung nach einer Stumpfbettung, die auch den anatomischen Begebenheiten der Amputierten Folge leistet, hat zu Änderungen in der Schaftkonstruktion geführt.

Die wichtigsten Argumente für eine sitzbeinumgreifende Einbettung des Oberschenkels im Vergleich zu einer sitzbeinunterstützenden Form sind:

- Keine Verdrängung der Muskulatur in m-I-Richtung; damit Erhalt der physiologischen Form des Oberschenkels - Keine Frontalpelotte zur Verhinderung des Abrutschens des Tuber os ischii von der Tuberbank mit Druckausübung im sog. „Scarpaschen Dreieck" damit auch keine Abschnürung der direkt unter der Haut liegenden Gefäße und Nerven (Arteria und Vena femoris, Nervus femoris)

- Physiologische Durchblutungssituation durch eine gleichmäßige Druckverteilung auf die gesamte Stumpfoberfläche

- Kein Auftreten beckenvorkippender Momente

- Keine Verdrängung der Sehne des M. adductor longus

- Keine Verdrängung der am Tuber ischiadicum ansetzenden Muskulatur (M. semitendinosus, M. semimembranosus, Teile des M. biceps femoris)

- Keine Inkongruenz zwischen Schaft und Tuber ischiadicum in der prothesenseitigen Schrittvorlage

- Kein „Wandern“ des Tubers auf der Tuberbank in m-I-Richtung (,Shifting“) durch die mediale Anlage am Ramus os ischii

- Zentrierung der Bodenreaktionskräfte auf das Hüftgelenk durch die Verklammerung vom Ramus os ischii und dem Trochanter major.

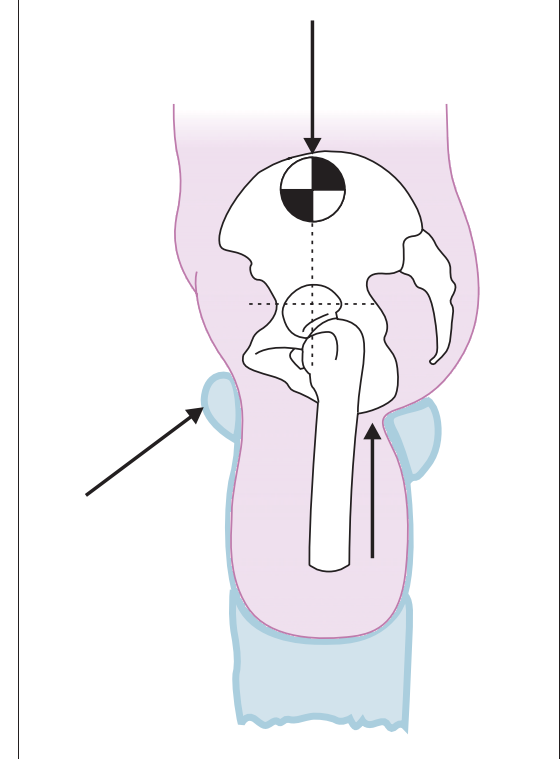

Abb. 17 Beckenvorkippendes Drehmoment durch Tuberunterstützung (Bufa-Archiv).

\section{Der Steuerungsbereich}

Wie schon bei der herkömmlichen Schaftform nötig, so kann auch beim längsovalen Schaft der Steuerungsbereich seine Aufgabe nur durch die Modellierung einer lateralen Anlage oder einer Femurspange, die die Adduktionsstellung des Femurs gewährleistet, erfüllen.

\section{Der Stumpfendbereich}

Der Kontaktschaft gilt heute als Standardversorgung und nur unter schwerwiegenden Gründen wird davon abgewichen. Die gesamte Stumpfoberfläche befindet sich im direkten Kontakt mit dem Prothesenschaft. Die Umsetzung zum Erhalt des hydrostatischen Systems ist nur so ohne Schädigung des Stumpfendes möglich. Die Ausbreitungsmöglichkeit für das Stumpfvolumen am Stumpfende(Schwellneigung mit Ödemgefahr) wird begrenzt.

Vorteile des Stumpfendkontaktes:

- Durch den Kontakt des Stumpfendes vergrößert sich die tragende Fläche, der Druck pro Flächeneinheit wird kleiner.

- Der intermittierende Druck (Druckschwankungen) fördert die Durchblutung und unterstützt die insuffiziente Muskelpumpe.

- Ein Bodengefühl kann entstehen (verbesserte Propriozeption)

- Die Schweißbildung ist geringer als in einem Schaft ohne Endkontakt

- Vermeidung von Ödemen 


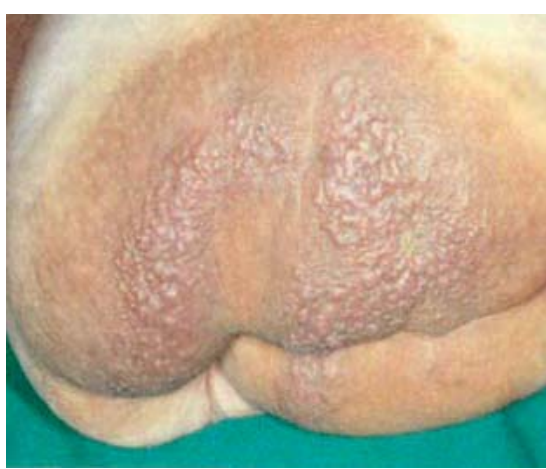

Abb. 18 Typische Veränderungen der Weichteilstruktur aufgrund eines nicht vorhandenen Stumpfendkontaktes im Schaft (TO Münster).

- Vermeidung von Verfärbungen und Hyperkeratosen (Abb. 18)

- Verminderte Neigung zu Phantomschmerzen.

\section{Prothetik nach Hüftexartikulation}

Einschneidende Veränderungen in der Weiterentwicklung dieser Prothesen in Hinblick auf die Einbettung, Materialund Aufbautechnik gab es 1954 durch eine Forschungsgruppe unter C. A. Mc Laurin vom Dept. of Veterans Affairs Canada. Diese Studien waren so grundlegender Natur, dass dieses System als „Kanadische Hüftexartikulationsprothese" seitdem auch im deutschsprachigen Raum bekannt ist und in Abwandlungen auch heute noch angewandt wird.

Dieser Prothesentyp hat im Verhältnis zu den bis dahin bekannten Systemen einige technische Vorteile:

- Das Hüftgelenk ist frei beweglich, lediglich gegen Überstreckung durch einen elastischen Anschlag gesichert

- Durch Verlagerung des prothetischen Hüftgelenkes in einem Winkel von $45^{\circ}$ nach vorn und unterhalb des natürlichen Gelenkes kann die Sitzfläche sehr flach gehalten werden

- Es entsteht keine Veränderung der Oberschenkellänge von der Standzur Sitzposition.

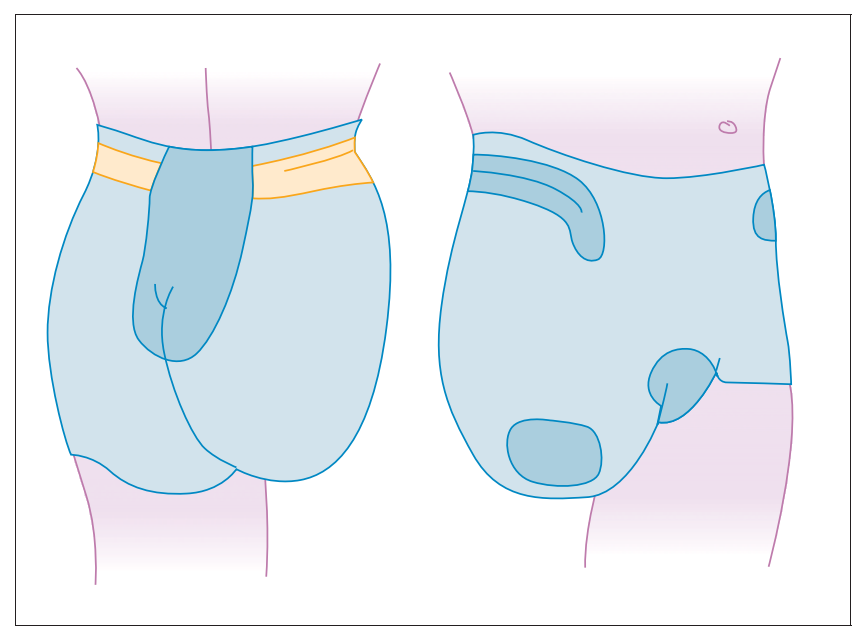

Abb. 19 Lastübertragung in einem Hüftexartikulationsschaft (Bufa-Archiv).

\section{Lastübertragung, Haftmechanismen und Führung der Prothese}

Wie bei jeder prothetischen Versorgung muss das gesamte Körpergewicht in der Standphase der Prothese auf diese übertragen werden. Diese Lastübertragung geschieht hauptsächlich über die Stumpfareale, die in Abb.19 gemustert gezeichnet sind. Die Haftmechanismen müssen in der Schwungphase der Prothese wirken, sodass diese am Körper fixiert bleibt. Dazu bietet sich in der Formgebung die Übergreifung der Hüftkämme an. Der Beckenschaft muss dementsprechend über die Hüftkämme reichen und dort so weit wie möglich in die Taille eingreifen. Bei der Hüftexartikulation werden alle, insbesondere aber die axialen Kräfte (Bodenreaktionskräfte) über die Weichteile auf die knöchernen Strukturen des Beckens übertragen (z.B. auf das Tuber ischiadicum). Bei der Hemipelvektomie müssen diese Kräfte auf die Weichteile (innere Organe) verteilt werden.

Zur Führung der Prothese darf der Beckenschaft nicht zu locker gefasst sein, insbesondere muss die a.-p. Weite (Abstand zwischen Kreuzbeinanlage und dem Bauch) einen flächenhaften Kontakt aufweisen, um die Bewegungen des Beckens in der Sagittalebene für die Fortbewegung nutzen zu können.

\section{Fazit}

Die unterschiedlichen, individuellen Formen und Stellungen von Stümpfen an der unteren Extremität erfordern vom Techniker ein umfassendes Bild bezüglich des Patienten. Es wird deutlich, dass der Abdruck am Patienten, das systematische Vorgehen in der Analyse der Passform und nicht zuletzt die Beachtung erforderlicher Aufbaukriterien noch immer Grundlagen sind zu einer individuellen Versorgung.

Nicht zuletzt zeigt sich außerdem, dass eine zufriedenstellende Versorgung im Sinne des Patienten nur in einem interdiziplinären Team möglich ist; angefangen vom dem das Hilfsmittel verordnenden Arzt über die physiotherapeutischen Maßnahmen bis hin zur technischen Versorgung.

\section{Literatur beim Verfasser.}

\section{Bernd Sibbel}

OMM, Fachlehrer

Detlev Kokegei

OMM, Fachlehrer

Bundesfachschule für

Orthopädie-Technik

Schliepstraße 6-8

44135 Dortmund 